\title{
HIGHLY EFFICIENT SPATIAL BEAM SPLITTER BASED ON COUPLED CAVITY WAVEGUIDE WITH SURFACE TAPER MODIFICATION
}

\author{
QUAN XU*, ${ }^{*}$, KANG XIE* $^{*}$, YIYING WANG $^{\dagger}$ and JUN TANG ${ }^{\dagger}$ \\ ${ }^{*}$ School of Optoelectronic Information \\ University of Electronic Science and Technology of China \\ Sichuan Province 610054, China \\ $\dagger$ School of Physical Electronics \\ University of Electronic Science and Technology of China \\ Sichuan Province 610054, China \\ ${ }^{\ddagger}$ xuquanwww@163.com
}

Received 19 November 2010

\begin{abstract}
A highly efficient spatial beam splitter based on coupled cavity waveguide with surface taper modification structure is proposed. The beam splitter is analyzed with the finite difference time domain numerical method. By modifying the number of the coupled multi-channel taper cylinders along horizontal direction and the number of waveguides along vertical direction, the spatial profile of the electric field can be varied and the direction of Poynting vector can be changed.
\end{abstract}

Keywords: Photonic crystal; coupled cavities waveguide; beam splitter.

OCIS Codes: 9999.9999, 250.5300, 230.1360, 230.3120

\section{Introduction}

The diffraction limit is perhaps the most elusive principle in optics. The light exiting from a region much smaller than half wavelength bears a strong angular spread that fills out the whole solid angle. ${ }^{1}$ Because of this, the highly directional emission has attracted attention in modern optical field in recent years. Most of those works fall into two categories: surface waves emitted by surface corrugations at the photonic crystal (PC) terminal ${ }^{2-4}$ and bulk waves emitted by multiples sources at the waveguide exit port. ${ }^{5}$

Recently, a novel photonic crystal waveguide has been reported. It is commonly referred to as a coupled cavity waveguide $(\mathrm{CCW})^{6-8}$ and consists of a chain of high $\mathrm{Q}$ optical cavities embedded in a PC. ${ }^{9}$ As a result of overlapping of the tightly confined modes, light propagation in $\mathrm{CCW}$ can be explained as photo hopping between nearby cavities. The most prominent feature of $\mathrm{CCW}$ is the feasibility of frequency tuning of its guiding modes. As changing the cavities very high rate of light transmission through sharp bends ${ }^{10}$ can be achieved, coupled cavity waveguides are a special optical component for integrated photonic 
circuits. Its special feature enables it to play an important role in overcoming the diffraction limit.

In this paper, a scheme to realize a highly efficient spatial beam splitter via CCW and surface taper multi-channel structure is proposed. The multi-channel of CCW can form a number of sources which interfere with each other. The effects of surface taper waveguide on transmission and emission angle are demonstrated. The taper surface can also improve the coupling between the emission surface of the photonic crystal and air. The effect of the number of CCW along the vertical direction and effect of the taper structure on the performance of the proposed splitter is discussed. Additionally, the optimum structure was shown to operate at the special wavelength suitable for optical communication.

\section{Description of the Design and Principle of Operation}

A two dimensional PC composed of square lattice of cylinders with refractive index of $n=3.4$ (corresponding to the dielectric constant of InGaAsP-InP semi-conductor material at wavelength of $1.55 \mu \mathrm{m}$ in vacuum) is considered. The radius of cylinder is $r=0.2 a$, where $a$ is the lattice constant of the PC. For TM polarization (electric field parallel to the cylinders), a wide band-gap exists in the normalized frequency range from $a / \lambda=0.29$ to $a / \lambda=0.422$ ( $\lambda$ is the wavelength in free space). The plane wave expansion method is used to calculate the band-gap.

A waveguide (W1) is constructed by removing one raw of the rods along $x$, as shown in Fig. 1(a). In Fig. 1(b), a CCW is created by removing some cylinders at regular interval of $2 a$ parallel to the $\mathrm{W} 1$, and a coupled multi-channel taper waveguide with $L_{x}$ (the number of cylinders along $x$ ) and $L_{y}$ (the number of waveguides along $y$ ) is added to the right side of the main structure. The radius of the cylinder decreases gradually from the left end to the right end of the taper zone from an initial value of $0.18 a$. Signal of continuous wave with a



(a)

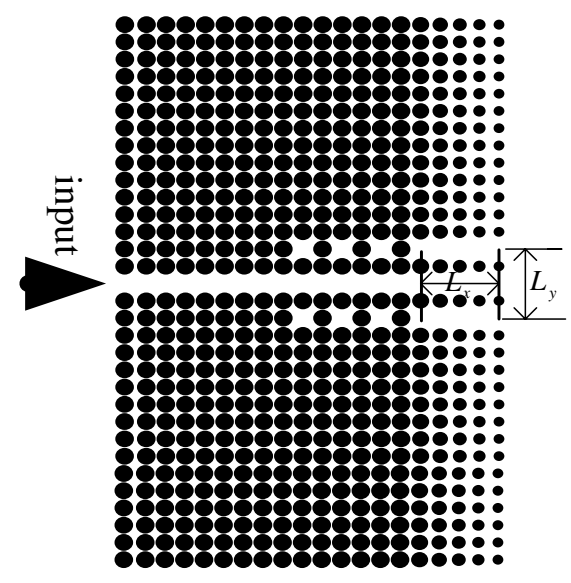

(b)

Fig. 1. The structure of waveguide. (a) Without CCW. (b) With CCW and $L_{x}=5, L_{y}=3$. 
normalized frequency of 0.408 is launched into the PC waveguide. To characterize the field of the output port, nineteen power detectors are placed along the arc shown in Fig. 1(a) with the interval of $5^{\circ}$. The distance between the output port of the $\mathrm{W} 1$ and the detectors is $10 a$. All results presented are for TM polarization and are obtained with the 2D FDTD with perfectly matched layer boundary conditions. ${ }^{11} 20 \times 20$ grids per unit cell are used in the simulations.

\section{Results and Discussion}

The results shown in Fig. 2 are calculated on the structure of Fig. 1(b) where CCW is inserted and the surface is modified. In order to vary the number of sources which interfere with each other, the number of CCW along vertical direction is changed while its influence on the number of sources is monitored. It is observed that the angular divergence of the output beam is significantly changed with the change of CCW. A $Y$ shaped spatial beam splitter with excellent performance and high transmission rate is achieved when $L_{x}=5$,

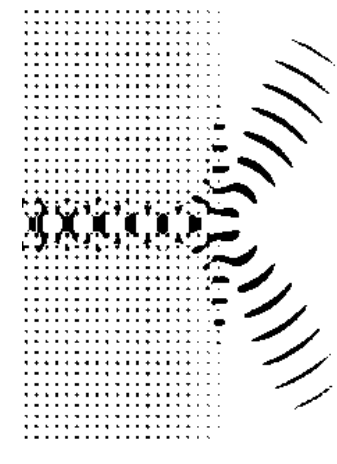

(a)

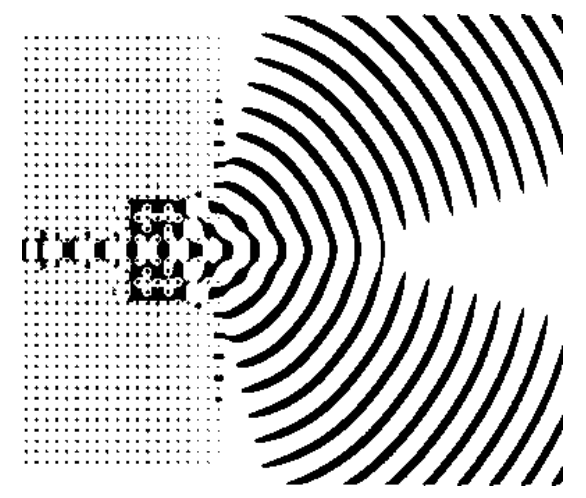

(c)

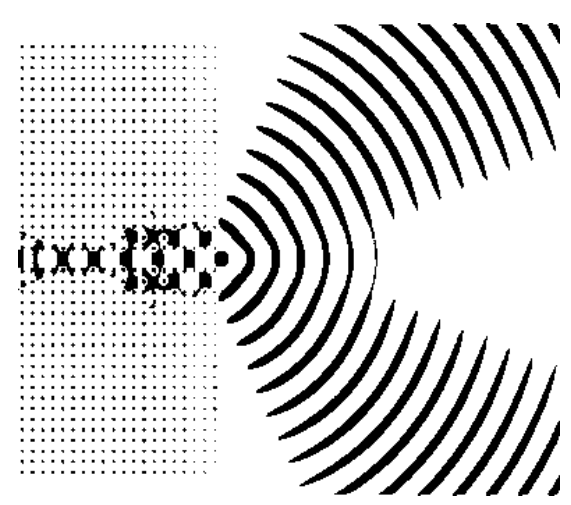

(b)

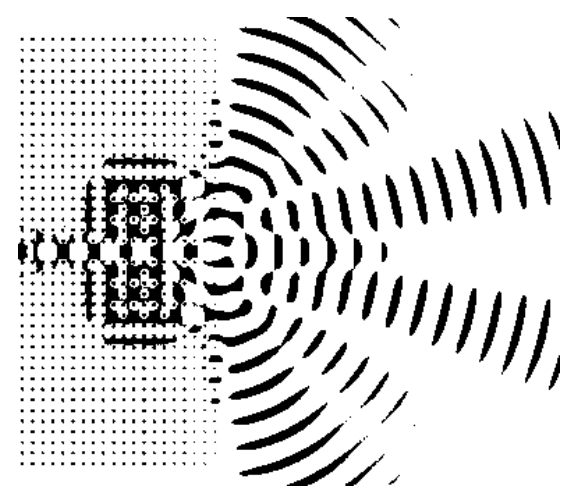

(d)

Fig. 2. Electric field distributions of the structure shown in Fig. 1(b) with $L_{x}=5$ and (a) $L_{y}=1$, (b) $L_{y}=3$, (c) $L_{y}=5$, (d) $L_{y}=7$. 


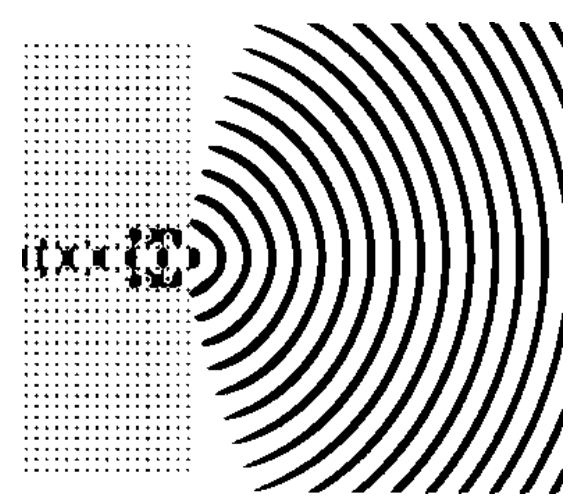

(a)

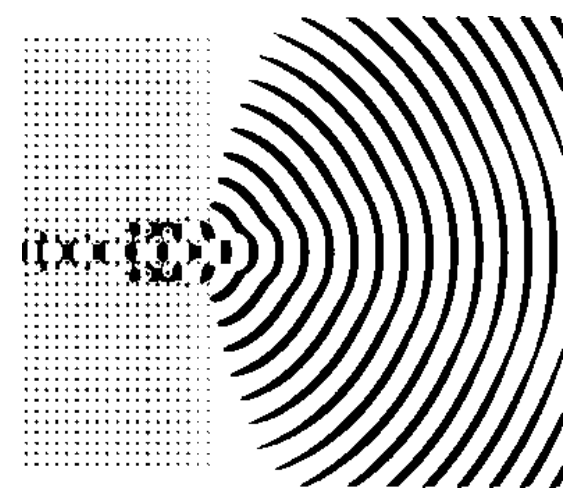

(c)

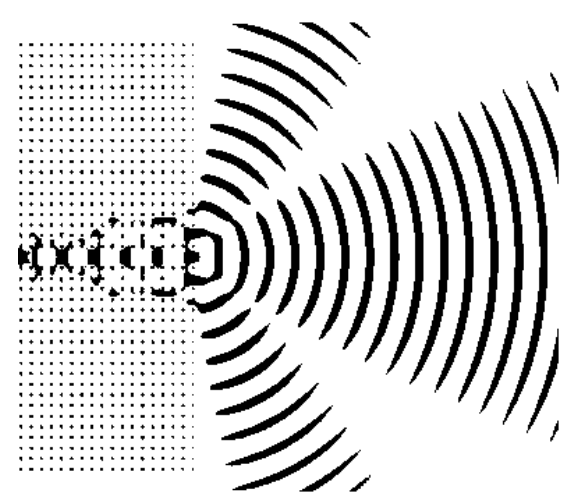

(b)

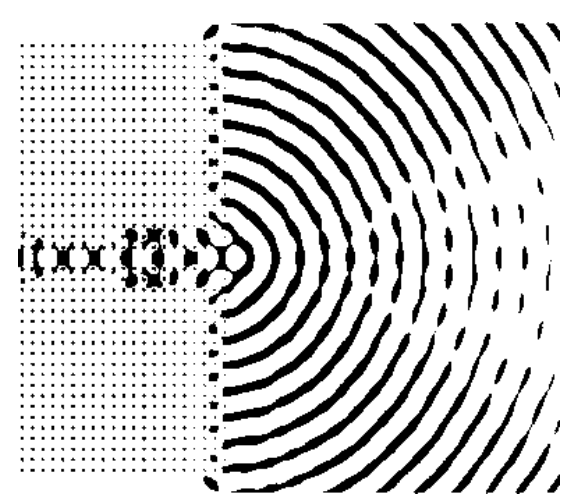

(d)

Fig. 3. Electric field distributions of structures of fixed $L_{y}=3$ and (a) $L_{x}=2$, (b) $L_{x}=3$, (c) $L_{x}=4$, (d) $L_{x}=6$.

$L_{y}=3$, as shown in Fig. 2(b). From the observation of Fig. 2, it is found that perfect $\mathrm{Y}$ beam splitter occurs at $L_{y}=3$. This agrees with the results predicted by the photonic crystal surface corruption theory.

Shown in Fig. 3 are the electric field distributions of structures of different $L_{x}$ with $L_{y}$ fixed at 3 . It is evident that the taper structure has a strong effect on the transmission rate and divergence angle of the emitting beam. The taper structures can obstruct the excitation of surface wave that enhances transmission along the vertical direction. By comparing Fig. 2(b) with Fig. 3, it can be derived that perfect spatial $\mathrm{Y}$ beam splitter prosperities are acquired by the structure at $L_{x}=5, L_{y}=3$. This is the suitable structure where the excitation of surface wave is obstructed almost completely, for the wavelength used.

The spectra of the emitting waves for different taper values are given in Fig. 4. From left to right, the radius of the cylinder in the taper zone decreases by $\Delta a$ from $0.18 a$. A continuous wave signal with normalized frequency of 0.408 is launched into the PC waveguide from the left. The spectra are obtained for three different values of $\Delta$. It is shown that $\Delta=0.02$ serves best the need of a $Y$-shaped beam splitter. 


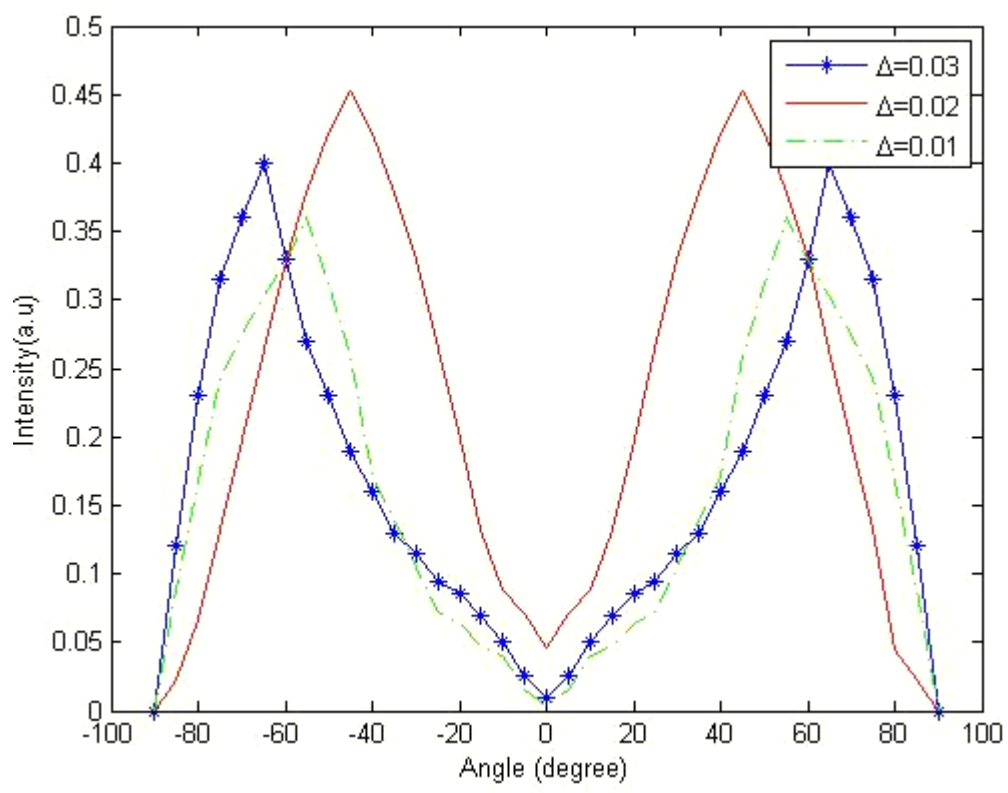

Fig. 4. Spectra obtained by the detectors for different taper values of $\Delta$.

The fabrication tolerance of the optimum structure can be assessed by launching waves of different frequency into the waveguide and investigating the outcome. In order to obtain this information, a Gaussian pulse with center frequency of 0.408 and bandwidth of 0.014 is launched into the device. A stable distribution of EM field is obtained in the output.

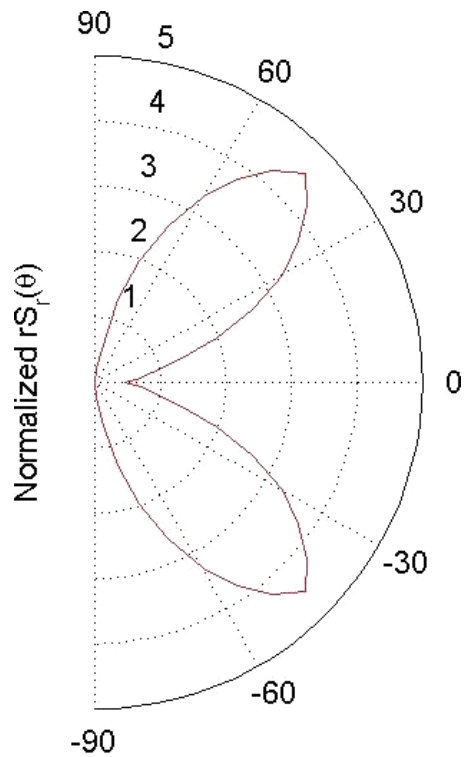

Fig. 5. Far-field pattern of the poynting vector $S_{r}$ of the structure shown in Fig. 1(b) with $L_{x}=5$ and $L_{y}=3$. 
The transmission rate predicted by FFT (fast fourier transfer) method is as high as $90.64 \%$ within the band. This is very good news for manufacturing purpose.

In order to gain a deeper insight into the propagation of signals in the optimum device, the Poynting vector $S_{r}$ as a function of azimuthal angle $\theta$ is calculated for the $Y$ shaped beam splitter for $L_{x}=5$ and $L_{y}=3$. The Poynting vector $S_{r}$ calculated by the 2D FDTD EM method is shown in Fig. 5. In the FDTD method, all the fields are obtained in the time domain. They need to be transformed into the frequency domain by the fast Fourier transform (FFT). This can be performed on the fly concurrently with the FDTD time stepping using the following formula,

$$
u(\omega)=\int_{0}^{N_{t}} u(t) e^{-i \omega t} d t \approx \sum_{n=0}^{N_{t}} u(n \triangle t) e^{-i n \omega \Delta t} \triangle t
$$

where $N_{t}$ is the total time steps, and $n$ is the current time step.

\section{Conclusion}

In conclusion, a highly transmissive spatial $\mathrm{Y}$ beam splitter is proposed. The impact of the coupled multi-channel taper waveguide on the performance of the splitter is discussed. The plane wave expansion is employed to calculate the band gap, and the FDTD method is used to analyze the distribution of the EM field of the structure. All those structures are found to have symmetrical energy distribution. While $L_{x}$ and $L_{y}$ are suitably chosen, high transmittance can be achieved. An optimum $Y$ shaped beam splitter with symmetrical energy distribution is designed with measured transmittance of $90.64 \%$. The enhancement on the transmittance of the light splitter may have potential applications in integrated optics and in micro-photonic circuits.

\section{Acknowledgment}

The author would like to acknowledge the financial support of the National Natural Science Foundation of China (NSFC) under Grant 60588502.

\section{References}

1. H. A. Bethe, Theory of diffraction by small holes, Phys. Rev. 66 (1944) 163.

2. E. Yablonovitch, Inhibited spontaneous emission in solid-state physics and electronics, Phys. Rev. Lett. 58 (1987) 2059-2062.

3. S. John, Strong localization of photons in certain disordered dielectric super-lattices, Phys. Rev. Lett. 58 (1987) 2486-2489.

4. K. M. Leung and Y. F. Liu, Full vector wave calculation of photonic band structures in facecentered-cubic dielectric media, Phys. Rev. Lett. 65 (1990) 2646.

5. Esteban Moreno, F. J. García-Vidal and L. Martín-Moreno, Enhanced transmission and beaming of light via photonic crystal surface modes, Phys. Rev. B. 69 (2004) 121402 (R).

6. Z. H. Zhu, W. M. Ye, J. R. Yuan and C. Zen, Enhanced transmission and directional emission via coupled resonator optical waveguides, Appl. Phys. B. 86 (2007) 327-331. 
7. A. Yariv, Y. Xu, R. K. Lee and A. Scherer, Coupled resonator optical waveguide: A proposal and analysis, Opt. Lett. 24 (1999) 711-713.

8. M. Bayindir, B. Temelkuran and E. Ozbay, Propagation of photons by hopping: A wave guiding mechanism through localized coupled cavities in three dimensional photonic crystal, Phys. Rev. B. 61 (2000) R11 855-R11858.

9. M. Bayindir, B. Temelkuran and E. Ozbay, Heavy photons at coupled cavity waveguide band edges in a three dimensional photonic crystal, Phys. Rev. B. 62 (2000) R2247-R2250.

10. M. Bayindir, B. Temelkuran and E. Ozbay, Tight-binding description of the coupled defect modes in three-dimensional photonic crystals, Phys. Rev. Lett. 84 (2004) 2140.

11. A. Mekis, J. C. Chen, I. Kurland, S. H. Fan, P. R. Villeneuve and J. D. Jiannopulos, High transmission through sharp bends in photonic crystal waveguides, Phys. Rev. Lett. 77 (1996) $3787-3790$.

12. Min Qiu, http://www.imit.kth.se/info/FOFU/PC/F2P/index.htm (2003). 